\title{
An Evaluation of Laboratory Specimen Rejection Rate in a North Indian Setting-A Cross-Sectional Study
}

\author{
${ }^{1}$ Dr.Abhineet Mehrotra, ${ }^{2}$ Dr. Kanchan Srivastava, ${ }^{3}$ Dr. Prabhakar Bais \\ ${ }^{I}$ Lecturer,Department of Microbiology,MRA Medical College, Ambedkar Nagar, UP, India \\ ${ }^{2}$ Scientist, DBT,Department of Pulmonary Medicine, KG Medical University, Lucknow, UP \\ ${ }^{3}$ Professor \& Head,Department of Biochemistry,KD Dental College, Mathura, UP, India
}

\begin{abstract}
Objective: To study the rate of specimens rejection received in the laboratory.
Design: A cross-sectional survey was conducted over a period of six months across Community Health Centres (CHC), Primary Health Centres (PHC), District hospitals and Govt. multi-specialty tertiary care hospital and trust autonomously run charity hospital. In-depth interviews were also conducted with the laboratory staff. Rejection of samples with the aim of proper processing of the samples and for providing report of high standard comes under good lab practices.
\end{abstract}

Results: A total of 2000 sample were studied and followed. A total of 5.3\% samples were rejected. The rejection rate was higher among the hospitals run by trusts than govt. In all, the rejection rate was higher blood sample (9.1\%) as compared to body fluid (8\%), urine (6.8\%), stool (5.3\%) and sputum (3.3\%) sample. The main reason of rejection was due to inadequacy of specimen collection by the paramedical staff.

Conclusion: The rejection rate was higher in trust hospitals due to higher awareness at the analytical level of the sample processing in the lab as compared to govt. run hospitals where every sample is processed irrespective of its adequacy/inadequacy and the report is provided. Thus, the emphasis should be given to make such diagnostic kits in future which are less dependent collection and handling.

Key Words: Specimen, Rejection rate, laboratory

\section{Introduction}

The laboratory in most cases has very little control on the collection of specimens for microbiological investigations. The education and awareness of the attendants, orderlies and nurses and attending physicians, who are involved in the collection and transport of the specimen to the laboratory is very important. On the other hand, the technicians must confer to the physician before rejecting any valuable specimens. Frequent staff discussions between the clinicians and the laboratory staff to bridge the gap is necessary to maintain appropriate quality control of lab findings.

In 2007 an article was published in Clinical Chemistry that compared the error rate of a stat laboratory in 1996 to the error rate in 2006. Although there was a significant reduction of errors, the distribution between preanalytical, analytical, and postanalytical phases of testing remained relatively consistent. Carraro and Plebani found that $87 \%$ of the errors occurred in the preanalytical phase, which includes proper patient and specimen identification, appropriate and correct test requests, accuracy in blood drawing, specimen handling, and specimen transportation. Furthermore, $73 \%$ of all the errors in all phases were classified as being preventable ${ }^{1}$.

Bonini et al, ${ }^{2}$ using the literature review approach, reported that a higher proportion of specimens that were collected at either inpatient or emergency department (ED) locations were rejected primarily because of hemolysis. The present study was conducted with the objective to find out the rate of rejection of the collected sample and to assess the attitude of staff involved in the collection of sample.

\section{Methodology}

A cross-sectional survey was conducted over a period of six months across Community Health Centres (CHC), Primary Health Centres (PHC), District hospitals and Govt. multi-specialty tertiary care hospital and trust autonomously run charity hospital. Around 2000 sample were studied for collection, handling and management of microbiology specimen where incorrect collection, mishandling and mismanagement were observed. Those sample were followed upto their final reports and clinical outcomes were correlated. For example, if sputum was not properly collected of a known clinically diagnosed case of tuberculosis, the outcome of the report and final treatment given by the clinicians under this scenario were also observed. A total of 30 (3 from each hospital) in-depth interviews was conducted from the lab technicians working in the hospitals. An informed consent from head of the hospital and from technicians was taken before including in the study. The study was approved by the Ethical Committee of the Institute. Rejection of samples with the aim of proper processing of the samples and for providing report of high standard comes under good lab practices. 


\section{Standard for specimen collection}

The standards for specimen collection are described briefly below ${ }^{3}$ :

\section{Standard for sputum collection}

A sputum sample was obtained by expectoration in the early morning The patient should be carefully instructed as the type of sample required. It must be coughed up from deep down in the chest Direct examination should be done as early as possible in order to avoid overgrowth by commensally organism present in the mouth.

\section{Mycobacterium Tuberculosis}

To detect Mycobacterium Tuberculosis in the sputum, it is important to ensure that the specimen does consist of purulent secretions coughed up from the bronchi (not saliva spot from the mouth). Collect the first sputum of the morning, if the sputum cannot be processed within 30 minutes, refrigerate the specimen and make smear and plate within three hours but no longer. If the specimen has to be transported use the transport media (NACL $1000 \mathrm{ml}$ with catylpyridinium chloride $10 \mathrm{mg}$ ).

\section{Precaution to be taken while making smear}

Proper ventilation should be in place where container of the specimen is opened and processed or it should be opened and processed near an open window and technician should wear mask and gloves so as not to infect himself and others as well.

\section{Urine Collection Standard}

Urine sample is meaningful only when the sample is a freshly voided first morning sample, since it is more concentrated and also formed elements are less likely to disintegrate. Examination of urine within $2 \mathrm{hrs}$ of collection is required because urine on standing at RT(room temperature) becomes alkaline and consequently distorts the RBCs and disintegrates WBCs and the casts. If the patient is confined to bed, collect the urine in a clean bedpan or urinal and transfer it at once to a regular container for urine collection and process the specimen promptly for lab tests. If the sample is for lab culture, it must be collected in a sterile container. Normally, the urine container should be not less than $300 \mathrm{ml}$ capacity. For routine and microscopic examination some labs use reusable containers which should be cleaned rinsed thoroughly in tap water and then in distilled water, drained and dried the presence of residual contaminants like detergents, disinfectants or a previous urine specimen, will lead to erroneous results. During the collection of urine in a female patient, vaginal discharge should be avoided. Urine collected during menstrual period should be so labeled or avoided for that time. Directly voided sample of urine can be used for routine urine analysis but not for microbiological examination. It requires patient preparation(female patients are advised to cleanse the area around the urethral opening with clean water, dry the area and collect the urine with the labia held apart and male patients are advised to clean hands before collecting the specimen) and the specimen is collected by the "clean catch" or "midstream"(middle of the urine flow) method in a sterile container.

\section{Blood collection standards}

Adult blood can be collected easily from any vein in both the arms, if not accessible try the wrist vein or veins from the foot of the legs. In pediatric patients, try carefully using smaller needle and in patients of few days to few months use femoral vein to draw blood. For serology testing, like IgG and IgM, and hormones serum should be separated carefully so that they are not hemolysed. Blood should be processed in the limited time for PCR.

\section{Blood culture}

For blood culture, the amount of blood collected (for adult $10 \mathrm{ml}$ and $1-5 \mathrm{ml}$ for pediatric patients) is diluted into at least 10 times its volume with a liquid medium like nutrient broth. Thus, a bottle must have at least $50 \mathrm{ml}$ of broth for inoculating $5 \mathrm{ml}$ of the specimen. Blood collection bottles can be monophasic with one type of media or can be biphasic with two type of media in the same bottle -liquid and solid. A portion of the blood specimen should also be put into thyoglycolate broth tube for the cultivation of any anaerobes present.

\section{Collection and handling of body fluid standards}

All body fluids (ascetic, pleural, CSF, synovial etc.) should be collected by a expert physician or trained nurse. Usually 2-3 tubes are taken for fluid collection. The second or third tubes are taken for microbiology study. The collection tube for microbiology study must without centrifugation. If yeast like organism are seen, part of the specimen is taken for the culture of cryptococcus. After that rest of the specimen is centrifuged (3000 rpm for $15 \mathrm{~min}$ and the sediment is used for the study of a stained smear, and for culture of 
the infectious agents CSF should be reported and never be refrigerated. The pathogenic organism will die, cells will lyse, and the glucose concentration will fall in standing CSF specimens.

\section{Stool collection standard}

Stool specimen for the laboratory can be collected in any type of cleaned disposable container either in the ward or at home. If the collection is done at home, patient must be clearly instructed not to collect the specimen in waxed paper or paper of any sort, or in small jars or bottle from which the specimen would be difficult to remove. Some tests would require preparations for the patients before the collection of the stool. The patient should not receive iron or other metallic compounds for 4-6 days before the collection of the specimen. For the examination of intestinal parasites, patients should not be under medication for at least 2 weeks before the specimen collection. It will be ideal to write these instructions and hand over to the patient prior to the collection of stool. Collection of specimen in the hospital or laboratory is more desirable than to attempt to collect a suitable specimen at home. A morning specimen before the breakfast is most desirable and should be processed by the lab within 2-3 hrs after discharge(worm stool), or else specimen can be collected at any time and should be processed promptly. Always collect the specimen without the admixture of urine. If possible have the patient urinate first.

The descriptive statistics are presented in proportions.

\section{Results}

A total of 2000 sample were studied and followed. A total of $45 \%$ were sputum, $30 \%$ were urine, $15 \%$ were stool $1.3 \%$ were body and $8.8 \%$ were blood samples from various type of hospitals (Table-1).

A total of 5.3\% samples were rejected. The rejection rate was higher among TMSTH than GMSTH (8.5\%), DH (6.3\%), CHC (2.3\%) and PHC (2.1\%) and signifying that percentage of appropriate sample comes in these CHC, PHC and DH are higher than trust run hospitals. In all, the rejection rate was higher blood sample (9.1\%) as compared to body fluid (8\%), urine (6.8\%), stool (5.3\%) and sputum (3.3\%) sample (Table-2).

It was observed that at PHCs, CHCs and even district hospitals none of the standard procedures was followed. Patient was even given a non sterile container, was not explained properly or half explained about only morning sample needed, and how to expectorate. Even when the sample was brought back, it was not processed in the prescribed time as of course there were many more to come and were processed after collecting all the specimen, but was not refrigerated as in some hospitals refrigerator wasn't there and in others if present not in working condition and even in working condition then no availability of electricity. When advised to the technician to follow proper procedure of collection and handling, there came the prompt reply that as there are so many patients to give sputum here(PHCs especially), time for counseling each of them is not possible. Same we came across in other govt. hospitals. Some of the technicians had developed an attitude towards not giving correct advise of collection and even not knowing correctly by themselves due to lack of proper training. But due to inappropriate collection and mishandling when incorrect reports are made especially of giving AFB positive patients AFB negative, leads to not proper treatment and these patients later on not only spread the infection to their family but some of them end up with MDR TB.

Problems were present when blood from rural areas were collected for HIV, HbsAg, HCV. In many cases, the collection were inappropriate and samples were hemolysed, and hence rejected for testing. Sample for blood culture were not collected in both containers (aerobic and anaerobic) and hence a partial report was handed over. Even containers were found to be contaminated prior to blood collection in it.

In govt. hospitals, many times CSF samples are not centrifuged before smear is made and culture is done and even CSF microscopy is reported as late as after 2-4 hrs, as sample was not provided to pathologist due to other lab works of the technician. When asked why not to follow the guidelines, they told that we manage somehow how so ever the specimen i s, and above all the stool specimen as they say do not carry much for diagnosis and clinician irrespective anything found in stool or not subscribe patient with all the relevant drugs be it antibiotic or antiprotozoal or antihelminth.

\section{Discussion}

The present study was conducted a cross-sectional with the primary objective of identifying the proportions of different specimen that were rejected by the point of collection. We found an overall specimen rejection rate of 5.3\% among various types of hospitals which was higher than a study in which the rejection rate among blood sample was $0.74 \%{ }^{4}$. The difference in the present study and other study might be due to hospital environment and technical staff. However, many national and international programs to track laboratory quality have reported laboratory specimen rejection rates ranging from $0.3 \%$ in outpatient facilities to $0.83 \%$ in hospital based laboratories ${ }^{5}$. 
The role of clinical pathology and laboratory medicine continued to grow as the single largest component of objective scientific data within the medical record of patients. The result of any laboratory examination is only as good as the sample received in the laboratory. Some specimens are time-dependent. In order for the laboratory departments to process them correctly, specimens must be collected/received within their time constraints to be accepted by the Laboratory. The Laboratory Manual should be consulted for specimen time limitations ${ }^{6}$.

The main reason of rejection was due to inadequacy of specimen collection by the paramedical staff. Some of the technicians had developed an attitude towards not giving correct advise of collection and even not knowing correctly by themselves due to lack of proper training. But due to inappropriate collection and mishandling when incorrect reports are made especially of giving AFB positive patients AFB negative, leads to not proper treatment and these patients later on not only spread the infection to their family but some of them end up with MDR TB.

\section{Conclusion}

The rejection rate was higher in trust hospitals due to higher awareness at the analytical level of the sample processing in the lab as compared to govt. run hospitals where every sample is processed irrespective of its adequacy/inadequacy and the report is provided. Thus, the emphasis should be given to make such diagnostic kits in future which are less dependent collection and handling.

\section{References}

[1]. Carraro P, Plebani M. Errors in a stat laboratory: Types and frequencies 10 years later. Clin Chem.2007;53:1338-134

[2]. Bonini P, Plebani M, Ceriotti F, Rubboli F. Errors in laboratory medicine. Clin Chem. 2002;48:691-695.

[3]. American Association for Clinical Chemistry. Expert Access-Dedicated Phlebotomy Improves Patient Care in an ED Setting. Fantz C. Nov 11, 2010.

[4]. Azadeh Stark, Bruce A. Jones, Deborah Chapman, JD, Karen Well, Richard Krajenta, Frederick A. Meier, Richard J. Zarbo, MD. Clinical Laboratory Specimen Rejection-Association With the Site of Patient Care and Patients' Characteristics. Arch Pathol Lab Med. 2007;131:588-592.

[5]. Dale JC, Novis DA. Outpatient phlebotomy success and reasons for specimenrejection: a Q-Probes study. Arch Pathol Lab Med. 2002; 126:416-419.

[6]. Arch Pathol Lab Med. 2007 Apr; 131(4):588-92.

Table-1: Distribution of sample collected in different hospitals

\begin{tabular}{|c|c|c|c|c|c|c|}
\hline Specimen & PHC & CHC & DH & GMSTH & TMSTH & $\begin{array}{c}\text { Total } \\
\text { Specimen }\end{array}$ \\
\hline Sputum & $\begin{array}{c}400 \\
(64.0)\end{array}$ & $\begin{array}{c}200 \\
(50.0)\end{array}$ & $\begin{array}{c}150 \\
(50.0)\end{array}$ & $\begin{array}{c}75 \\
(22.1)\end{array}$ & $\begin{array}{c}75 \\
(22.4)\end{array}$ & $\begin{array}{c}900 \\
(45.0)\end{array}$ \\
\hline Urine & $\begin{array}{c}125 \\
(20.0)\end{array}$ & $\begin{array}{c}125 \\
(31.3)\end{array}$ & $\begin{array}{c}50 \\
(16.7)\end{array}$ & $\begin{array}{c}150 \\
(44.1)\end{array}$ & $\begin{array}{c}150 \\
(44.8)\end{array}$ & $\begin{array}{c}600 \\
(30.0)\end{array}$ \\
\hline Stool & $\begin{array}{c}100 \\
(16.0)\end{array}$ & $\begin{array}{c}75 \\
(18.8)\end{array}$ & $\begin{array}{c}25 \\
(8.3))\end{array}$ & $\begin{array}{c}50 \\
(14.7)\end{array}$ & $\begin{array}{c}50 \\
(14.9)\end{array}$ & $\begin{array}{c}300 \\
(15.0)\end{array}$ \\
\hline $\begin{array}{l}\text { Body fluid including SF, } \\
\text { ascetic, pleural etc }\end{array}$ & - & - & - & $\begin{array}{c}15 \\
(4.4)\end{array}$ & $\begin{array}{c}10 \\
(3.0)\end{array}$ & $\begin{array}{c}25 \\
(1.3)\end{array}$ \\
\hline $\begin{array}{l}\text { Blood (for serology and } \\
\text { Culture etc) }\end{array}$ & - & - & $\begin{array}{c}75 \\
(25.0)\end{array}$ & $\begin{array}{c}50 \\
(14.7)\end{array}$ & $\begin{array}{c}50 \\
(14.9)\end{array}$ & $\begin{array}{l}175 \\
(8.8)\end{array}$ \\
\hline Total & $\begin{array}{c}625 \\
(100.0)\end{array}$ & $\begin{array}{c}400 \\
(100.0)\end{array}$ & $\begin{array}{c}300 \\
(100.0)\end{array}$ & $\begin{array}{c}340 \\
(100.0)\end{array}$ & $\begin{array}{c}335 \\
(100.0)\end{array}$ & $\begin{array}{c}2000 \\
(100.0)\end{array}$ \\
\hline
\end{tabular}

PHC-Primary Health Centre, CHC-Community Health Centre, DH-District Hospital, GMTH-Government owned Multi-specialty Tertiary care Hospital, TMTH-Trust run Multi-Specialty Tertiary care Hospital, values in the parentheses are the percentage 
Table-2: Rejection rate of the collected sample

\begin{tabular}{|l|c|c|c|c|c|c|}
\hline Specimen & PHC & CHC & DH & GMSTH & TMSTH & $\begin{array}{c}\text { Total } \\
\text { Specimen }\end{array}$ \\
\hline Sputum & $\begin{array}{c}8 \\
(2.0)\end{array}$ & $\begin{array}{c}4 \\
(2.0)\end{array}$ & $\begin{array}{c}2 \\
(1.3)\end{array}$ & $\begin{array}{c}7 \\
(9.3)\end{array}$ & $\begin{array}{c}9 \\
(12.0)\end{array}$ & $\begin{array}{c}30 \\
(3.3)\end{array}$ \\
\hline Urine & 3 & 4 & 7 & 12 & 15 & 41 \\
$(2.4)$ & $(3.2)$ & $(14.0)$ & $(8.0)$ & $(10.0)$ & $(6.8)$ \\
\hline Stool & 2 & 1 & 4 & 4 & 5 & 16 \\
Body fluid including SF, & $(2.0)$ & $(1.3)$ & $(16.0)$ & $(8.0)$ & $(10.0)$ & $(5.3)$ \\
ascetic, pleural etc & -- & -- & -- & 1 & 1 & 2 \\
\hline Blood (for serology and & -- & -- & 6 & $\begin{array}{c}5 \\
(6.7)\end{array}$ & $\begin{array}{c}5 \\
(10.0)\end{array}$ & $\begin{array}{c}5 \\
(8.0)\end{array}$ \\
\hline Culture etc) & 13 & 9 & 19 & 29 & 35 & $(10.0)$ \\
Total & $(2.1)$ & $(2.3)$ & $(6.3)$ & $(8.5)$ & $(10.4)$ & $(5.3)$ \\
\hline
\end{tabular}

PHC-Primary Health Centre, CHC-Community Health Centre, DH-District Hospital, GMTH-Government owned Multi-specialty Tertiary care Hospital, TMTH-Trust run Multi-Specialty Tertiary care Hospital, values in the parentheses are the percentage of respective sample collected 